\title{
Science, daily practice and education: different sides of the same coin
}

\section{G. Campanelli ${ }^{1}$}

Published online: 22 September 2020

(c) Springer-Verlag France SAS, part of Springer Nature 2020

One of the major criticisms of inguinal and abdominal wall surgery is the widespread tendency to add personal modifications to well-established technical approaches. Accordingly, Lichtenstein, Lap IPOM and retromuscular repair, to cite just some examples, are often interpreted in so many different ways that it becomes impossible to compare the results of their application and, ultimately, to provide clear and safe recommendations on the indications for each technique.

While some specialists might condemn such behavior as impossible or even crazy, in the real world, it is extremely common among surgeons, notwithstanding the many and widespread systems and opportunities that are available to those wanting to learn and to keep abreast of the scientific literature.

Consequently, a clear gap has opened up between the "World of Science" and the world of routine surgical work done in departments the world over.

As a result of this situation, the education received by residents varies hugely across the different settings, and so too, therefore, does the treatment received by patients.

And while this state of affairs might be the norm in less developed countries, it seems astonishing, from a technical point of view, that such differences and gaps in the education of residents should persist in more developed settings.
However, there does exist a way of overcoming these difficulties, thanks to the role that can be played by major scientific societies and leading scientific journals and publications.

Ad hoc schools organized by international societies, e-learning provided by serious scientific groups, and constant reading of rigorous and globally recognized scientific journals appear to be the essential ingredients of the solution.

In line with this, I firmly believe that young residents and assistants among our own readers can benefit greatly from our journal, to update their knowledge and ultimately their approaches to patients.

Only in this way, can each professional seriously evaluate and compare his/her own results, and on the basis of this evaluation, ensure that every single patient receives optimal treatment.

Publisher's Note Springer Nature remains neutral with regard to jurisdictional claims in published maps and institutional affiliations.
G. Campanelli

hernia.editorialoffice@gmail.com

1 University of Insubria, Gruppo Ospedaliero San Donato, Milan, Italy 